\title{
Post-exercise changes in energy intake using current methodology are not reproducible in overweight and obese women
}

\author{
G. L. Brown, M. E. Lean and C. R. Hankey \\ Life-Course Nutrition and Health, Centre for Population and Health Sciences, University of Glasgow, Glasgow G4 OSF, UK
}

Direct observation(s) of energy intake via buffet meals served in the laboratory are often carried out within short-term exercise intervention studies. Reproducibility of energy intake values assessed using this method in lean males at rest is high ${ }^{(1,2,3)}$, but the reproducibility of values obtained has not been assessed either under resting control conditions or post-exercise in overweight and obese females.

Fourteen sedentary, overweight and obese, pre-menopausal females completed four trials; two exercise and two control, following a randomised, counterbalanced design. Each trial lasted $24 \mathrm{~h}$ spanning over $2 \mathrm{~d}$; afternoon on day 1 and morning on day 2 . An exercise session to expend $1.65 \mathrm{MJ}$ was completed on day 1 of exercise trials, subjects remained sedentary during control trials, and observation while sedentary was continued for a further $5 \mathrm{~h}$ on day 2 of all trials. Three ad libitum buffet meals were served during each trial to assess energy intake; evening meal on day 1 and breakfast and lunch on day 2. Appetite was continuously assessed during trials using a visual analogue scale. Paired $t$-tests were used to assess differences between mean values obtained from the control and exercise trials. Reproducibility of energy and macronutrient intake values and appetite ratings was assessed by intraclass correlation coefficient $\left(r_{\mathrm{i}}\right)$.

There were no differences in energy intake between the control $(10.4(0.6) v .9 .2(0.7) ; P=0.08)$ and exercise trials $(10.7(0.6) v$. $10.3(0.7) ; P=0.62$ ) (mean (SEM)). There were no differences in macronutrient intake between exercise trials, protein and fat intake were significantly different between control trials $(P<0.02)$. Values of $r_{\mathrm{i}}$ were significant for energy intake in control trials but not exercise trials. Reproducibility of macronutrient intake and appetite ratings was poor; $r_{\mathrm{i}}$ values for all measurements are presented in the table.

The $r_{\mathrm{i}}$ values for $24 \mathrm{~h}$ macronutrient and energy intake after a single aerobic exercise session are not significant; hence, these measurements are not reproducible. The $r_{\mathrm{i}}$ values for total energy, protein and fat intake in control trials were significant with large associated CI, indicating a large degree of individual variation. The $r_{\mathrm{i}}$ values for appetite ratings were largely significant in control and exercise trials, but all had large CI. The present findings indicate that the laboratory-based buffet meal method of assessing energy intake does not provide reproducible results in sedentary, pre-menopausal overweight and obese women either under control or exercise conditions.

\begin{tabular}{|c|c|c|c|c|}
\hline & Control trials & $95 \% \mathrm{CI}$ & Exercise trials & $95 \% \mathrm{CI}$ \\
\hline \multicolumn{5}{|l|}{ Food intake } \\
\hline Energy intake (MJ) & 0.50 & $0.03,0.80 *$ & 0.04 & $-0.53,0.55$ \\
\hline Carbohydrate intake (g) & 0.16 & $-0.31,0.60$ & 0.08 & $-0.45,0.57$ \\
\hline Protein intake $(\mathrm{g})$ & 0.42 & $-0.08,0.77^{*}$ & -0.09 & $-0.50,0.42$ \\
\hline Fat intake $(\mathrm{g})$ & 0.50 & $0.003,0.81 *$ & -0.06 & $-0.50,0.44$ \\
\hline \multicolumn{5}{|l|}{ Appetite } \\
\hline Hunger & 0.29 & $-0.23,0.69$ & 0.65 & $0.20,0.87^{*}$ \\
\hline Satiety & 0.56 & $0.07,0.83 *$ & 0.44 & $-0.07,0.77 *$ \\
\hline Fullness & 0.71 & $0.30,0.90 *$ & 0.31 & $-0.23,0.71$ \\
\hline PFC & 0.40 & $-0.17,0.76$ & 0.54 & $0.07,0.82 *$ \\
\hline DTE & 0.43 & $-0.09,0.77^{*}$ & 0.58 & $0.08,0.85^{*}$ \\
\hline
\end{tabular}

Values are mean $95 \% \mathrm{CI}, * P<0.05$

1. Gregersen NT, Flint A, Bitz C et al. (2008) Am J Clin Nutr 87, 1277-1281.

2. Nair NS, Brennan IM, Little TJ et al. (2009) Br J Nutr 101, 1094-1102.

3. Arvaniti K, Richard D \& Tremblay A (2000) Br J Nutr 83, 489-495. 